\title{
SEXUAL SELECTION AS A CONSEQUENCE OF PATHOGEN AVOIDANCE BEHAVIORS
}

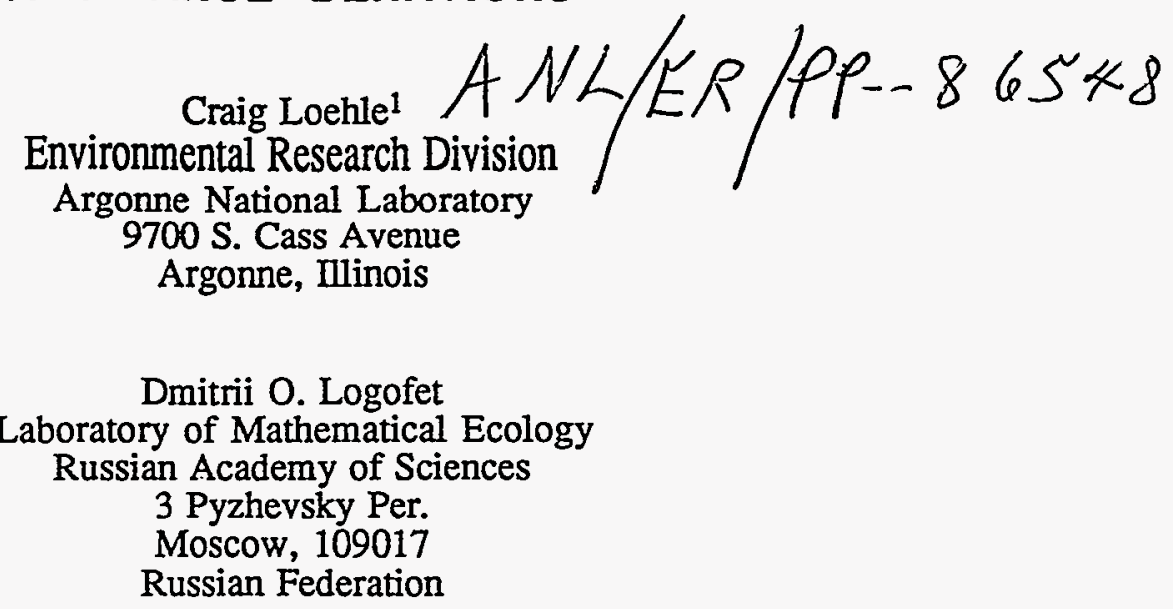

\section{ABSTRACT}

The current theory that sexual selection results from female choice for good genes suffers from several problems. An alternative explanation is proposed. The pathogen avoidance hypothesis argues that the primary function of showy traits is to provide a reliable signal of current disease status so that sick individuals may be avoided during mating. Our studies shown that a significant risk of pathogen transmission occurs during mating and that showy traits are reliable indicators of current disease status. The origin of female choosiness is argued to lie in a general tendency to avoid sick individuals, even in the absence of showy traits. The showy traits are argued to originate as simple exaggerations of normal traits that are indicative of good health (bright feathers; vigorous movement; large size). Thus the origins of both showy traits and female choosiness are not problematic in this theory. A game theory analysis is employed to formalize the theory. Results of the game theory model support the theory. In particular, when the possession of male showy traits does not help reduce disease in the female, then showy traits are unlikely to occur. This case corresponds to the situation in large flocks or herds in which every animal is thoroughly exposed to all group pathogens on average. Such species do not exhibit showy traits. The good genes model does not make this prediction. The pathogen avoidance model can also lead to the evolution of showy traits even when selection is not effective against a given pathogen (e.g., when there is no heritable variation for resistance) but will lead to selection for resistance if such genes are present. Overall, the pathogen avoidance hypothesis provides a complete alternative to the good genes theory.

1 Corresponding author. 


\section{DISCLAMIER}

Portions of this document may be illegible in electronic image products. Images are produced from the best available original document. 


\section{INTRODUCTION}

One of the great conundrums in evolutionary theory is posed by the existence of secondary sex traits. That these traits do not contribute directly to survival and may even increase mortality was clear even to Darwin and caused him considerable discomfort. While several theories have been put forward to explain the existence of these traits, all of the theories have substantial unresolved problems. Loehle (1995) argued that whereas secondary sex traits are not necessarily reliable indicators of fitness, they are reliable indicators of current disease status. If a female uses these traits to avoid contamination during mating or during nest-keeping (or both), then she will obtain an advantage in fitness. In this study, we extend and formalize this insight by using game theoretical models.

\section{Extant Models of Sexual Selection}

The basic model of sexual selection is the good genes theory. The theory that females just happen to'prefer bright males for no reason is simply not tenable, and we will not consider it further.

Before considering the good genes theory, we need to distinguish two types of traits: contest-related traits are those traits that are used directly in male contests for females but are not necessarily involved in female choice. In deer, the antlers are used for pushing and wrestling contests. The winner gets the females. In elephant seals, size is selected for during fights for territory. The female does not choose in either of these cases. We thus exclude such secondary traits from our model because they are rather simply explained as an outcome of contest competition. The other type of secondary trait includes singing displays, bright feathers or other markings, long tail feathers or crests, wattles, dances, bowers, and offers of food. These traits are the types we will be referring to as "showy" traits. These traits are influential in systems in which females choose a mate or can reject a suitor. 
The dominant theory purporting to explain the origin of sexual selection (Hamilton and Zuk, 1982) posits a link between traits for ornamentation and genes for parasite resistance (the good genes hypothesis). This theory points in the right direction, but Loehle (1995) argues that it is current level of health (lack of contagion) that females select for, not necessarily good genes.

There are several problems with the good genes theory. First, secondary sex traits do not provide a reliable indicator of fitness or even of the possession of genes for pathogen resistance (Kirkpatrick, 1986, 1989; Loehle, 1995; Read, 1988). Second, one must make rather strong and arbitrary assumptions about genetic linkages between genes for pathogen resistance and secondary sex traits in order to get the selection process started. In particular, one must have females who happen to have a preference for a particular unusual "showy" trait at the time when this trait first occurs, but even this unusual circumstance is not sufficient because when both traits are rare, the choosy female and the "showy" male are not even likely to encounter each other and will thus be at a disadvantage. Third, if sexual selection were effective in selecting for genes for pathogen resistance, we should expect more brightly colored species to have fewer parasites, whereas the opposite trend holds. Fourth, because most species suffer adversely and often catastrophically from parasites and disease, it is worth asking why more species do not exhibit bright coloration or other displays. In particular, there are trends in the type of species that do not show these traits consistent with a pathogen avoidance explanation. Fifth, it is problematic why females should exhibit any preference for an initially rare and unusual trait. Again this poses a problem for initiating the process.

\section{THE PATHOGEN AVOIDANCE HYPOTHESIS}

Let us consider now the alternative hypothesis that sexual selection acts to reduce pathogen transmission. By this theory, secondary sex traits are reliable flags or markers by which the female (usually) can determine which individuals are diseased so that she can avoid them. Several conditions must be met for this theory to hold: (1) A significant risk of pathogen transmission 
must occur during mating. (2) Secondary sex traits must be reliable indicators of current disease status. (3) The fact that females are usually choosier must be explained. (4) The types of species exhibiting these traits must be explained. (5) Selection models must be consistent with the hypothesis. We explore each of these five conditions in turn.

Although disease transmission, especially during mating, has not been studied well, enough data exist to posit a significant risk to most animals. Mating is a route for transmission of sexually transmitted diseases (e.g., simian HIV), regular diseases, skin parasites, and yeast and fungal infections, both to the partner and to the offspring (Service, 1986). Brucellosis (Witter, 1981) and myxomatosis (Yuill, 1981) may be transmitted by mating and may kill directly or cause sterility. Rabies may also be transmitted by biting during mating encounters. In rats, prevention of male grooming after copulation leads to a high incidence of transmission of genital infections (Hart and Haugen, 1971; Hart et al., 1987). Hart (1990) argues that postcopulatory autogrooming in the genital area evolved for prevention of disease and notes that species that exhibit such behavior show fewer sexually transmitted diseases than those that do not. Thus, mating clearly poses a significant risk that deserves more thorough study.

The second criterion is that secondary sex traits must provide a reliable indicator of current disease status. Displays and mating dances are clearly affected by the health of the male (e.g., in parasitized guppies [Kennedy et al., 1987]). Physical signals have also been shown to be affected by health. Rätti et al. (1993) showed that pied flycatchers infected with Trypanosoma had shorter tails and shorter wings and arrived at the breeding grounds on average two days later, with a consequent reduction in breeding success. Bower provisioning will be affected by individual vigor. Antler and body size in deer have been shown to be adversely affected by liver fluke infestation (Mulvey and Aho, 1993). Illness can affect the luster of the fur in dogs. Other effects are summarized by Hill (1995). The scant data available suggest that females will avoid diseased males, even in the absence of secondary sex trait "flags" (e.g., Edwards and Barnard, 1987; 
Kennedy et al., 1987). Edwards (1988) found that healthy mice direct increased exploratory behaviors (e.g., sniffing) toward infected mice but exhibit less contact. Loehle (1995) documented other avoidance behaviors.

These observations support the concept that infections (particularly if severe) affect secondary sex traits. Further, the observations suggest a mechanism by which the sexual selection process may get started. If a general tendency exists to avoid ill individuals, as documented by Loehle (1995), and if an ability to avoid them during mating exists in the absence of secondary sex traits, then this provides a basic female behavior of avoidance that will be preexistent. This set of preexistent behaviors overcomes the difficulty in the good genes model of why the female should exhibit a preference initially to get the selection process started. This avoidance behavior also solves a second difficulty, which is why particular traits come to be preferred. We may simply say that almost all secondary sex traits (especially when incipient) represent an exaggeration of traits exhibited by healthy individuals: vigorous movements, bright colors, loud singing, etc. Females thus exhibit a natural preference for such traits because they amplify the signal of "healthiness." This model further explains the arbitrariness of the traits: any random exaggeration of normal signs of health and vigor will provide a reliable signal of disease status and can be amplified by being favored.

We must next explain why females are choosier in general. The standard explanation is that females invest more energy in a smaller number of young, an observation that is certainly true. The implication is that females should be more picky about the gene quality of their partners; however, females also are at more risk from disease physiologically than are males. Because female reproductive structures are internal, they are subject to diseases that do not affect males (e.g., yeast and chlamydia infections in humans). Further, autogrooming after mating is less effective in females for the same reason. Although males often get exhausted during the breeding season, females face a longer period of stress while gravid or pregnant and while raising the 
young. Sickness during this period poses a substantial risk. In addition, if the female passes the disease or parasites on to the young, they will suffer inordinately because of their weakness. For these reasons, we would expect females to be far more averse to risk of disease than males.

We also must explain the types of species that are likely to exhibit these traits. Because most species are likely to be at some risk for disease and parasites, it is an anomaly that only certain species exhibit such traits. The disease avoidance model provides an explanation. For choosiness to be beneficial, the selection of a disease-free mate must reduce the risk of disease. If, however, the animal typically lives in very large social groups, then each animal is largely exposed to all of the pathogens of the entire group, in which case extra choosiness is not beneficial. Birds of this type (gulls, penguins, starlings, and geese) never exhibit extreme secondary sex traits. In herding mammals, secondary sex traits are associated with male contests (bison; wildebeest), not female choice. The good genes theory does not predict this pattern. The disease transmission model, by contrast, specifically predicts that secondary "showy" sex traits will be almost exclusively confined to species in which mating (and nest-keeping) are primary modes of pathogen transmission, which is not the case for species living in large groups. The trait need not be confined to polygynous species and is, in fact, observed in some territorial songbirds.

The final criterion is the existence of a selection model consistent with the disease avoidance theory. This criterion represents the major part of this report and is covered in the next section.

To recap the features of the pathogen avoidance model, it predicts that showy traits should originate as arbitrary exaggerations of features indicative of health and vigor; that showy traits should be reliable indicators of current health, but not necessarily of the possession of good genes; and that species living in large social groups should not generally benefit from showy traits. The model further provides an explanation for why females should be more choosy. Note that female choosiness will provide not merely a relative fitness advantage but a population fitness advantage 
by reducing mortality. Further, choosiness will increase female fitness even in the absence of heritable variation for pathogen resistance (as occurs when a population is faced with a novel pathogen); that is, sexual selection in this model need not result in selection for good genes, although it will do so if such genes are present.

\section{Evolutionary Stable Strategies and Evolutionary Stable States}

What might be the evolutionary consequences of bearing showy traits as an indicator of health status can be analyzed along the lines of the game theory approach proposed by Maynard Smith (1974) to study the evolution of animal conflicts. We have reconciled the logic of his famous "hawks-doves" game and modified the payoff matrix of the game to make it applicable to competition for mating in a population of males with and without a showy trait. A variety of dynamic regimes is shown to be theoretically possible, including evolutionary stable strategies and evolutionary stable states; and which particular regime takes place in the model turns out to be determined by the relative magnitudes of the formal game parameters. The parameters are interpreted in terms of fitness and this interpretation suggests a way of reasoning on whether and how particular phenomena in the biology or mating ethology of an animal species may or may not promote selection for showy traits.

Following the basic ideas of the game-theoretical theory of evolution (Hofbauer and Sigmund, 1988), we suppose a single-species population to consist of $n$ phenotypes that are labeled by $1,2, \ldots, n$ and are identified with certain (pure or mixed) strategies played by the phenotypes in a conflict. If we denote by $x_{1}, x_{2}, \ldots, x_{n}$ the frequencies of the phenotypes in the population $\left(x_{1}+x_{2}+\ldots+x_{n}=1\right)$, then the phenotypic structure, or composition, of the population is represented by the $n$-dimensional vector $x=\left[x_{1}, x_{2}, \ldots, x_{n}\right]$ belonging to the standard simplex $S_{n}$ of the $n$-dimensional linear space. 
Because genetic determination of a strategy is generally not known, we will not try to reproduce in equations the "Mendelian machinery of inheritance" (Hofbauer and Sigmund, 1988, p. 124) but rather assume that the rate of increase $x_{i}^{\prime} / x_{i}$ of the phenotype $i$ 's portion in the composition is a measure of its evolutionary success, which, in its turn, may be expressed as the difference between the fitness $f_{i}(x)$ of phenotype $i$ and the average fitness

$$
f_{\mathrm{av}}(x)=x_{1} f_{1}(x)+\ldots+f_{i}(x) x_{i}+\ldots+x_{n} f_{n}(x)
$$

of the composition. This yields the game dynamical equation

$$
x_{i}^{\prime}=x_{i}\left[f_{i}(x)-f_{a v}(x)\right], \quad i=1, \ldots, n,
$$

for which the simplex $S_{n}$ is an invariant set: if $x\left(t_{0}\right) \in S_{n}$, then $x(t) \in S_{n}$ for all $t \geq t_{0}$.

The main idea of the game-theory approach is to identify the gain (or loss) in the phenotype fitness with the outcome of the underlying game, which is defined by the payoff matrix $U=\left[u_{i j}\right]$, $i, j=1,2, \ldots, m$. The entry $u_{i j}$ of the matrix represents an award (payoff) that the player of pure strategy $s_{i}$ gets when playing against the pure strategy $s_{j}$. For a mixed strategy $q=\left[q_{1}, \ldots, q_{m}\right] \in$ $S_{m}$, which is, by definition, to play strategy $s_{i}$ with probability $q_{i}$, the expected payoff is

$$
q_{1} u_{1 j}+q_{2} u_{2 j}+\ldots+q_{m} u_{m j}
$$

when $q$ is played against the pure strategy $s_{j}$; but if the opponent also applies a mixed strategy $p=\left[p_{1}, \ldots, p_{m}\right]$, then the expected payoff to the $q$-strategist (under the assumption of independent strategy choice) is equal to

$$
q_{1} u_{11} p_{1}+q_{2} u_{21} p_{1}+\ldots+q_{m} u_{m 1} p_{1}+
$$




$$
\begin{aligned}
& q_{1} u_{1 j} p_{j}+q_{2} u_{2 j} p_{j}+\ldots+q_{m} u_{m j} p_{j}+ \\
& \ldots \\
& q_{1} u_{1 m} p_{m}+q_{2} u_{2 m} p_{m}+\ldots+q_{m} u_{m m} p_{m}
\end{aligned}
$$

which, in the matrix form, is equivalent to $\langle q, U p\rangle$, the scalar product of vectors $q$ and $U p$.

In the population, let there be only two phenotypes, 1 and 2 , playing strategies $q$ and $p$ respectively and having frequencies $x_{1}$ and $x_{2}$. Because each of the phenotypes is playing "against the field" that responds by strategy $q$ with frequency $x_{1}$ and by strategy $p$ with frequency $x_{2}$, their expected payoffs are given by

$$
\begin{aligned}
& f_{1}(x)=<q, U\left(x_{1} q+x_{2} p\right)> \\
& f_{2}(x)=<p, U\left(x_{1} q+x_{2} p\right)>
\end{aligned}
$$

The change of variables $x_{1}=x, x_{2}=1-x$, reduces system (1.1) to a single equation:

$$
x^{\prime}=x\left[f_{1}(x)-f_{\mathrm{av}}(x)\right]=x(1-x)\left[f_{1}(x)-f_{2}(x)\right]
$$

(with the prime sign ( ) designating the derivative with respect to time), for which simplex $S_{2}=\{x$ : $0 \leq x \leq 1\}$ is the invariant set.

Note that if, in the (mathematically) degenerated case, $f_{1} \equiv f_{2}$ everywhere in $S_{2}$, which biologically means that the phenotypes are actually identical in the aspects under study, then any particular composition $\left[x_{1}, x_{2}\right]$ is a neutrally stable equilibrium in Eq. (1.3); that is, it remains unchanged unless a perturbation (external with respect to the equation) changes the composition. This implication is logical from the general form of Eq. (1.1), which, by design, is a frequencydependent, rather than density-dependent, model of microevolution (Pohley and Thomas, 1983). 
In general, the equation says nothing about whether the population survives or perishes, but it does describe the dynamics of the relative population composition under the selection factors considered and under the assumption (implicitly made) that factors unconsidered affect the phenotypes equally well. To the extent that these assumptions are true, Eq. (1.1) and its further implications, like Eq. (1.3) and subsequent ones, represent an appropriate tool to investigate the evolutionary consequences of particular phenomena in the species ethology, like those mentioned previously.

Note also that both the general Eq. (1.1) and its particular form Eq. (1.3) depend on the difference in the fitness functions, rather than on their absolute values. This observation means that all of the functions can be combined with an arbitrary function, $g(x)$, thus allowing the model to be combined with another model of microevolution that might take into account other factors of population dynamics.

With regard to Eq. (1.3), the spectrum of dynamical behaviors of its solutions (trajectories) is not too rich. In particular, Eq. (1.3) lacks chaos and periodical regimes, so that trajectories tend eventually' to one or another rest point, or equilibrium. Given the fitness functions $f_{1}$ and $f_{2}$ defined on $S_{2}$, a standard isocline analysis can fix the number and stability properties of the equilibria, which appear to depend exclusively on the qualitative pattern in the mutual arrangement of the fitness curves. Table 1 summarizes a variety of such patterns (the first column), indicating their dynamical consequences (columns 2 through 4). Qualitatively, the outcome is determined by the number of intersections in the fitness curves and the sign of the difference $f_{1}-f_{2}$ near the intersection. We have confined the depiction to no more than two intersections, the cases $1^{\prime}$ and $4^{\prime}$ being not included, which yield the same picture as 1 and 4 after the permutation of the boundary points and subscripts; artificial exotic cases of curve tangency are also excluded. The outcome does not depend on the curvatures of the fitness curves, which may actually be straight lines.

\section{DISCLAIMER}

This report was prepared as an account of work sponsored by an agency of the United States Government. Neither the United States Government nor any agency thereof, nor any of their employees, makes any warranty, express or implied, or assumes any legal liability or responsibility for the accuracy, completeness, or usefulness of any information, apparatus, product, or process disclosed, or represents that its use would not infringe privately owned rights. Reference herein to any specific commercial product, process, or service by trade name, trademark, manufacturer, or otherwise does not necessarily constitute or imply its endorsement, recommendation, or favoring by the United States Government or any agency thereof. The views and opinions of authors expressed herein do not necessarily state or reflect those of the United States Government or any agency thereof. 
The point, however, is whether and how any of these qualitative types of dynamical behavior can be realized via the payoff matrix representation (1.2) for the fitness functions, which reduces Eq. (1.3) to

$$
x^{\prime}=x(1-x)[x<p-q, U(p-q)>-<p-q, U p>] .
$$

When either or both of the boundary rest points, $x=0$ and $x=1$, of Eq. (1.3) are at least locally Lyapunov stable (as in any row of Table 1 except the second one), speaking of evolutionary stable strategies fixed in the population makes sense. If, indeed, the point $x=0$ is stable, the population consists entirely of $p$-phenotype individuals, while any small deviation from this point vanishes with time (i.e, any small portion $\varepsilon$ of the $q$-phenotypes is eliminated from the population). Thus, a population of $p$-strategists cannot be invaded by $q$-phenotypes, and this idea is the basic idea behind the formal notion of the evolutionary stable strategy (ESS).

Definition 1. A strategy $p$ is said to be evolutionary stable if for all $q \in S_{m}, q \neq p$, the inequality

$$
<q, U(\varepsilon q+(1-\varepsilon) p)><<p, U(\varepsilon q+(1-\varepsilon) p)>
$$

holds true for all $\varepsilon>0$ that are sufficiently small (Hofbauer and Sigmund, 1988).

The explicit sense of inequality (1.5) is that the $p$-phenotype fares against the mixture of $q$ and $p$-strategists better than any other $q$-phenotype. The implicit sense of the inequality is that, for Eq. (1.4), the inequality implies the stability conditions to hold for the rest point $x=0$ (Hofbauer and Sigmund, 1988). The next to last column of Table 1 indicates which one of the two phenotypes (if any) plays the ESS for the corresponding fitness pattern. 
However, if neither $x=0$ nor $x=1$ is dynamically stable, it means that there is no evolutionary stable phenotype. If, on the other hand, a nontrivial equilibrium $x=x^{*}\left(0<x^{*}<1\right)$ turns out to be dynamically stable, it means that both phenotypes coexist in the population, which now represents a stable mixture of strategies $p$ and $q$ (i.e., a "mixed" strategy again); however, to be rigorous, we should not recognize $x=x^{*}$ as an ESS because it is no longer associated with either particular phenotype. Rather, $x=x^{*}$ is an evolutionary stable state of the population (which - abbreviates unfortunately to "ESS" again) or an evolutionary stable composition (ESC) of the population phenotype structure.

From the mathematical point of view, no reason exists to oppose the existence of ESSs to that of ESC: depending on formal parameters of the game, both may or may not coexist in a model [Eq. (1.3)] (see the last column of Table 1). In subsequent particular examples, even with linear fitness functions, we see various kinds of combinations of the existence and (local or global) stability of the rest point $x=x^{*}$ with stability of $x=0$ and $x=1$, which gives certain grounds to speculations on evolutionary effects of particular phenomena in the biology or sex ethology of the species.

\section{"Humble-Showy" Competition as an Evolutionary Game}

To analyze evolutionary consequences of the hypothesis that a showy trait gives a male an advantage in mating, we simplify the spectrum of mating behaviors in an animal population to the existence of only two phenotypes, one possessing the trait and another one without it. The former is conventionally called "showy," while the latter is called "humble"; and the assumption is made that "showy" males always win the competition for a female, whether it occurs as a breeding tournament (in the form of "demonstration") or a female's choice (as in females visiting male bowers). This assumption is the major hypothesis of the model. In terms of strategies, phenotype 1 always plays the (pure in this sense) strategy of bearing the showy trait, while phenotype 2 
always plays the (not less "pure") strategy of lacking the showy trait. In the notation of the previous section, we have $q=[1,0]$ and $p=[0,1]$, whereby the fitness functions $(1.2)$ reduce to

$$
\begin{gathered}
f_{1}=(U[x, 1-x])_{1}=u_{11} x+u_{12}(1-x), \\
f_{2}=(U[x, 1-x])_{2}=u_{21} x+u_{22}(1-x) .
\end{gathered}
$$

If we assumed the "handicap" hypothesis that those carrying the showy trait must be very fit to be able to afford the show, then the analysis would be no more than a confirmation of the trivial logic that it is "better to be rich and healthy than poor and ill." On the contrary, we assume that the showy trait is to be paid for in terms of individual fitness to other-than-mating aspects of the life cycle (e.g., escaping predation, surviving migrational stresses). We therefore express this cost of the show as a generalized parameter $S>0$, representing the loss in fitness of the "showy" phenotype compared with the "humble" one.

Similarly, we evaluate successful mating in terms of another generalized parameter, $G$, which is associated with the per capita reproductive potential of the phenotype-specific males. Winning the competition for a female should result in a reproduction success, hence a gain, $G>0$, in the fitness of the "showy" phenotype when the loser is "humble," and a gain twice as small, $G / 2$, when the loser, who fails in reproduction, is "showy," too.

When the "humble" phenotype fails in contest with the "showy" one, it fails to reproduce; hence the gain in its fitness should be zero. A nonzero gain, $\mathrm{H}>0$, obtains, yet is shared by two, when the "humble" individual gets the chance of reproduction by winning a contest with another "humble" one. If we deal with a population where "humble" individuals that are mated with are more likely to be sick than the "showy" ones, purely because the female can better distinguish if a "showy" individual is sick, then mating with a "humble" has a higher risk of disease transmission to the female or offspring (or both), and the "humble" phenotype can invest less in raising the 
young than the "showy". In this case mating with a "humble" individual results in poorer survival of offspring, and we have to assume that $H<G$. Other population phenomena may imply other relationships.

Thus, neglecting the cost of competition itself, we come to the following payoff matrix for the outcome of the contest:

\begin{tabular}{l|ll} 
& showy & humble \\
\hline showy & $-S+G / 2$ & $-S+G$ \\
humble & 0 & $H / 2$
\end{tabular}

which may also be designated as:

$$
U=\left[u_{i j}\right]=\left[\begin{array}{cc}
-S+G / 2 & -S+G \\
0 & H / 2
\end{array}\right]
$$

with nonnegative parameters $H, G$, and $S$.

From the general fact that it is the difference between the fitness functions, rather than their absolute values, that is important to Eq. (1.1) and its corollaries, it follows (Hofbauer and Sigmund, 1988) that adding any values $c_{j}$ to any $j$-th column of the payoff matrix does not change Eq. (1.4). This reasoning justifies the highly abstracted meanings of the previous parameters, of which the relative values only (relative to each other) may be of any real sense. 
Fitness functions (2.1) take on the following particular forms:

$$
\begin{gathered}
f_{1}(x)=G-S-G x / 2, \\
f_{2}(x)=(1-x) H / 2,
\end{gathered}
$$

and represent straight lines unless the parameters themselves vary with $x$.

In contrast to the hawk-dove game (Maynard Smith 1974, 1982), the "humble-showy" game has now three, rather than two, formal parameters, which may not however be absolutely independent if we make additional assumptions, such as $H<G$. It is not surprising therefore that, whereas the former model or similar models have no ESS (Hofbauer and Sigmund, 1988), the latter does have it in particular domains of the three-dimensional parameter space. Mathematically, the existence or nonexistence of an ESS in a model ensues from the dynamical behavior of its trajectories. Thus, in what follows, we present the outcome of stability analysis of Eq. (1.3) with the fitness' functions (2.3), rather than by mere verification of the formal ESS criterion (1.5), which certainly does or does not hold in the relevant cases.

The variety of fitness patterns reported in Table 1 is specified further in Table 2 for the model (1.3) with the linear fitness functions (2.3). The diversity of patterns is almost entirely realized by straight lines (2.3), with the clear exception of case 4 . [If two straight lines intersected at two points, they would actually coincide, meaning mathematically that any point $x$ of $S_{2}$ is a neutral equilibrium and, biologically, that no difference exists among the phenotypes with respect to the selection factors under study. Mathematical conditions of the coincidence can be easily obtained from (2.3); they represent the equality-type relations between the formal parameters and are normally neglected in theoretical analysis as too "artificial" and having zero probability to hold in any real situation.] 
Formal conditions that characterize the fitness patterns are presented in the second column of Table 2 as a pair of general inequalities that define the pattern. The solution for the particular case of linear fitness functions (2.3) determines the region in the positive octant of the three-dimensional parameter space $(G, H, S)$ where the pattern is realized, with the corresponding outcome of the model dynamics. The last column of Table 2 gives graphic images for the regions, each being designed rigorously as a part of the space restricted either by the coordinate planes or - the corresponding (infinite) petals of the two planes, $S=G-H / 2$ and $S=G / 2$, intersecting along the straight line at the (untraced) plane $G=H$. The images again emphasize the idea that, although the pattern is defined exclusively by the parameter values relative to each other, certain relations are of importance even between the relative values.

Straightforward interpretations ensuing from the biological sense of the formal parameters of the humble-showy competition game are given subsequently for each of the cases considered. In Case 1, for example, with the "showy" fitness function always dominating over the "humble" one, the domination takes place when $G>H / 2$ and if

$$
S<\min \{G-H / 2, G / 2\}
$$

whereby the "showy" ESS is even globally stable, and any small portion of "showy" phenotypes will not only fix in the population but will also exclude all of the "humble" competitors. This exclusion is not surprising if the reproductive "cost of the show" is really not too high. More surprising is that the reproductive gain of the "showy" winning the contest with the "humble" may be even less than the gain of the "humble" defeating another "humble" (but greater than half the gain).

On the contrary, when the cost of the show is sufficiently high:

$$
S>\max \{G-H / 2, G / 2\}
$$


(case 1), the "humble" phenotype represents a globally stable ESS, so that any fraction of the population consisting of showy individuals is doomed to extinction regardless of the $H$ and $G$ values relative to each other.

Intermediate values of the cost $S$ lead to other fitness patterns depending on the "level of intermediacy" relative to the other two parameters. In particular, the inequality

$$
G / 2<S<G-H / 2,
$$

which makes sense only when $G>H$, leads to case 2 . The latter condition means that the benefit of the "showy" winning the contest with the "humble" should be greater than the "humble" defeating another "humble." The former condition then means that the cost of the show takes on moderate values that are in a certain balance with the reproductive gains of both phenotypes. These conditions deprive both boundary points of stability (so that none of the phenotypes is an ESS) but guarantee the existence and global stability of the ESC as the nontrivial equilibrium state

$$
x^{*}=[2(\mathrm{G}-S)-H] /(G-H) \text {. }
$$

Logically, the lower the cost of the show and the greater the difference between the "showy's" and the "humble's" gains, the higher is the "showy" portion in the ESC.

Case 3 arises when the inequality

$$
G-H / 2<S<G / 2
$$

holds true, which itself makes sense only when $G<H$. The latter means that the gain of the "showy" should be less than that of the "humble." Condition (2.8) then means that the cost of the show takes on moderate values again comparable with the reproductive gains. In this situation, 
each of the phenotypes represents an ESS, while the one that is realized should be the one that is more abundant relative to $x^{*}$. The point of (unstable now) equilibrium

$$
x^{*}=[H-2(G-S)] /(H-G)
$$

divides the interval $(0,1)$ into the two corresponding intervals of attraction (see Figure 1 ). It is logical again that the greater the difference between the "showy's" gain and the cost of the show and the smaller the "humble's" gain, the smaller is $\mathrm{x}^{*}$; and hence, the wider is the "showy" interval of attraction (i.e. the higher is the chance that the type fixed will be the "showy" ESS).

Note, however, that the case $G<H$ is contrary to our hypothesis that females choosing a "showy" male should reduce the risk of disease to themselves or their offspring (or both). The only reason "showy" represents an ESS here is that "showy" males always win the contest against "humble" males. This case therefore corresponds to sexual selection purely on the basis of female choice (for no reason) for a secondary sex trait; that is, the females prefer the "showy" males even though these males produce a smaller fitness outcome for the females. We view this case as implausible. This case also corresponds to the contest competition case in which the males who win the contest (such as rutting bighorn sheep) actually sire fewer offspring (in the limit, the male that wins the harem is impotent).

\section{Female Choosiness}

Because the sexual selection process involves both a male showiness trait and a female choosiness trait, examining the evolutionary game from the female's point of view is also useful. Rather than performing a full game analysis, it is sufficient here to simply look at the payoff table parameters from the standpoint of female choice. Two cases are relevant, where the female can discriminate disease by means of showy traits and where she can not. The former case implies that the current status of the trait (e.g. the presence or absence of aberrations in the showy plumage of 
birds [Hamilton and Zuk, 1982]), is an indicator of the disease status. The latter case corresponds to the situation when the animal lives in large social groups (the female is already exposed to pathogens, and mating does not increase risk) or when secondary sex traits do not provide any information on disease status (e.g., early-stage syphilis or AIDS).

If females can discriminate between sick and healthy individuals (by means of the showy .trait), then the fitness gain of the "showy" males should always be greater than that of the "humble" ones $\left(G_{\mathrm{d}}>H_{\mathrm{d}}\right)$ because females avoid pathogen transmission to themselves or offspring when preferring "showy" males. To what particular extent $G_{\mathbb{d}}$ is greater than $H_{\mathrm{d}}$ is to be determined by the actual level of pathogen-induced mortality among the male offspring or actual decrease in their viability caused by shortage of nest care when the male is sick. Which particular case of those listed in Table 2 is then realized is determined further by the value of $S_{\mathrm{d}}$, the cost of the show, relative to $G_{\mathrm{d}}$ and $H_{\mathrm{d}}$.

However, if females cannot discriminate between sick and healthy individuals at all, then mating with either type gives no benefit in terms of avoiding pathogens. The relation between $G$ and $H$ is to be determined again by the reasons external to our model, for instance, by whether or not bearing the showy trait is itself strongly correlated with the disease status; however, bearing no additional hypotheses in mind, we have to presume that $G_{\text {nd }} \approx H_{\text {nd }}$ (i.e., there is no direct linkage between "showiness" and fitness). Because the showy trait is the one that makes discrimination possible, mating with "humble" males should not reveal any benefit from discrimination. Thus we have also to presume that $H_{\mathrm{nd}} \approx H_{\mathrm{d}}$.

Now, by means of model (1.3) and (2.1-2), we can compare evolutionary consequences of females being and not being choosy, yet only in terms of selection for a showy trait. By assuming the cost of the show being, though arbitrarily small or large but, fixed and equal in both situations, we can trace, from inspecting the conditions of Table 2 , the effect that female choosiness exerts on the "showy-humble" microevolution. Table 3 represents a report from this kind of inspection of 
the particular cases where, in accordance with the previous logic, the formal parameters of the game are given with the following particular values relative to the lowest gain, $H_{0}>0$, in the "humble" fitness:

$$
G_{\mathrm{nd}}=H_{\mathrm{nd}}=H_{0}=H_{\mathrm{d}}<G_{\mathrm{d}}=2 H_{0} \text { or } G_{\mathrm{d}}=4 H_{0}
$$

The pure theoretical state of nondiscrimination, $G_{\mathrm{nd}}=H_{\mathrm{nd}}=H_{0}$, reveals only cases 1 and $1^{\prime}$ in model behavior, with cases 2 and 3 being generally impossible, yet degenerating at $S_{\mathrm{nd}}=0.5$ $H_{0}$ to the "no difference" situation mentioned in the previous section. To illustrate case 2 or 3 , we have to assume a nonempty range of difference existing between $G$ and $H$, which, however, should be small enough to meet the nondiscriminating assumption, $G_{\mathrm{nd}} \approx H_{\mathrm{nd}}$.

To have the whole range of parameter $S$ mathematically continuous we also had to investigate some boundary cases generating nonstrict inequalities in Table 3, whereas Table 2 gives them strict meanings only. As a matter of fact, the boundary (equality) conditions result in either nothing new or no more than emergence of the boundary equilibrium opposite to the existing ESS; because this opposite equilibrium is unstable, it does not change the "generic" picture of model behavior within the given subrange.

If we now fix the cost of the show at a low level, say, $S_{\mathrm{d}}=S_{\mathrm{nd}}=0.3 H_{0}$, which means the cost to be equivalent to $30 \%$ loss from the reproductive gain to the fitness, then we see that both the discriminating and nondiscriminating populations realize case 1 , where the "showy" is the unique ESS. However, when the cost exceeds $0.5 \mathrm{H}_{0}$, it already becomes "too expensive" to afford the show for the nondiscriminating populations, which now realize case $1^{\prime}$, where the "humble" is the unique ESS. The discriminating populations, on the contrary, still retain the "showy" as a unique ESS. Only further increase in the cost $S_{\mathrm{d}}$, up to $S_{\mathrm{d}}<1.5 H_{0}$ for $G=2 H_{0}$ and up to $S_{\mathrm{d}}<2.5 H_{0}$ for $G=4 H_{0}$, results in coexisting "humbles" and "showies" in the form of the globally stable ESC. Smaller values of $S_{\text {nd }}$ already extinguish the "showy" from the 
nondiscriminating populations, while only further increase beyond $S_{\mathrm{d}} \geq 1.5 H_{0}$ or $S_{\mathrm{d}} \geq 2.5 H_{0}$ brings about exclusion of the "showy" phenotype from the discriminating populations, too.

These particular observations can be mathematically generalized in the statement that the "showy" phenotype fixes in a discriminating population when

$$
0 \leq S_{\mathrm{d}}<G_{\mathrm{d}}-H_{0} / 2,
$$

and in a nondiscriminating population when

$$
0 \leq S_{\text {nd }}<G_{\text {nd }}-H_{0} / 2=H_{0} / 2 .
$$

Because discrimination has been identified fundamentally with the condition $G_{\mathrm{d}}>H_{\mathrm{d}}=H_{0}$, it follows that the range (3.1) is fundamentally wider than (3.2). Moreover, the greater the difference between $G_{\mathrm{d}}$ and $H_{\mathrm{d}}$ (i.e. the benefit from discrimination), the wider is the range of costs where the "showy," although existing steadily in the discriminating population, is already doomed to extinction in the nondiscriminating one. Note, however, that the condition in (3.2) only holds if we assume that "showy" males continue to win in contests with "humble" males, in spite of the lack of fitness gains to the female (i.e., that female choosiness is exhibited for no reason in the nondiscrimination case). In the absence of gain, however, female choosiness is likely to be extinguished, in which case the possibility of a "showy" ESS under nondiscrimination (case 3, Table 3) vanishes.

So, while increasing the cost of the show tends generally to eliminate the "showiness", the choosiness of females expands the range of costs where the "showy" is still evolutionary stable, thus strongly promoting selection for showy traits. Note that this conclusion has come from comparing two microevolutionary processes: one with female discriminating ability and another one without such discrimination. If we would like to study microevolution of the female 
choosiness trait itself in a population with "showy" and "humble" phenotypes, then we should have to consider (after adopting a certain sex ratio) a new model with the two female phenotypes, too, and the females' own payoff matrix. This consideration would lead us to a somewhat more complex mathematical apparatus called bimatrix games, which lie beyond the scope of the present report.

\section{DISCUSSION}

In the framework of a simple game-theory model (1-2.1) for species microevolution, we have analyzed the evolutionary consequences caused by a "strategy" of having a showy trait that provides for an advantage in mating yet costs something in terms of reproduction. The game is defined by three parameters, $G, H$, and $S$, which generate a certain pattern of the payoff matrix (2.2) and which, although being highly abstracted themselves, do allow one to speculate on the relative contribution that each makes to the outcome of microevolution. The analysis has revealed that the showy trait does provide for a selective advantage in the microevolution if the reproductive cost of the show, $S$, is not too high. If $S$ is sufficiently small, then the showy strategy is evolutionary stable even in the case where the per capita reproductive potential of the "showies," $G$, is less than that of the "nonshowies," $H$ (yet still $G>H / 2$ ). Increasing $S$ may turn this ESS, which means here the phenotypic homogeneity of the population, into a globally stable ESC, which means the stable coexistence of both phenotypes. This result differs from the predictions of good genes theories. A situation also exists with two opposite ESSs (when the parameters are approximately equal), with the ESS being realized that is closer to the initial composition. Naturally, a too-high cost of the show makes the nonshowy strategy evolutionary stable. Female choosiness (i.e., an ability to discriminate disease status and to avoid mating with sick males), is shown to promote selection for showy traits even though the showy trait may result in mating failure for its sick bearer at the individual level. If discrimination is not possible, showy traits are always more costly and hence more likely to be eliminated. 
These effects are the major, or "primary," ones that the formal parameters of the game have on the outcome of microevolution within a constant-matrix model. In using the abstract model in arguing on the role that showy traits (which are typical secondary sex traits) play in microevolution of a particular species, one has to relate the parameters to the relative contributions that the major population processes make to the population dynamics. If, for example, the species evolves under a strong predation pressure to which the showy individuals are more vulnerable, then the cost of the show cannot be regarded as small or moderate. Heavy parasite or pathogen load on the population, combined with a high probability of pathogen transmission during mating, should certainly increase the value of $G$ relative to that of $H$ if the female can avoid mating with sick males, and so forth.

The way of reasoning by which the relative values of the model parameters are inferred from the biology of a species should also give a hint on whether those values can be considered as constants, thereby yielding a model of the previous kind, or whether they should vary as the phenotypic composition changes; for example, if the showy trait strongly correlates with parasite resistance (i.e., if there is heritable variation in fitness), then the total parasite load should be a decreasing function of $x$, the frequency of the showy phenotype in the population; while both $G$ and $H$, the reproduction parameters, should be some functions of the load which increase when the load decreases. The payoff matrix (2.2) hereby becomes a function of the composition $x$, resulting in nonlinear fitness functions $f_{i}(x)$. As the diversity of the nonlinear fitness patterns is generally greater than that of the linear ones, the variety of dynamical regimes of the "primary" model may be expanded. The latter suggests that some "secondary" dynamic effects of the secondary sex traits might arise.

At this point, it is useful to list the specific assumptions and predictions of the theory and either compare them to existing data or discuss how they might be tested. 
1. Sexual selection is less likely to occur if female choosiness provides no reduction in the rate of pathogen transmission. This situation exists in large social groups where the individual is thoroughly exposed to all group pathogens. Showy traits should not occur in such species, a prediction supported by observations, as discussed. Also, for pathogens not transmitted by conspecifics, sexual selection will not be effective. Leeches and mosquitoes are classic examples. This contrasts with predictions of good genes theories which do not distinguish between transmissible and nontransmissible pathogens.

2. Sick individuals should be more effectively selected against during mating from among a group of showy individuals than from among a group of dull ("humble") individuals. In this case, we must be careful that a test of this prediction be based on genetically "showy" types versus genetically "humble" types and not just on phenotypes, because the level of health affects these traits directly. This prediction may be tested experimentally.

3. While increased fitness of offspring sired by showy males is predicted to occur (as in von Schantz et al., 1994) it is also predicted that cases will occur in which heritability of fitness linked to showy traits cannot be demonstrated, in contrast to the good genes hypothesis. These cases should occur where pathogen avoidance increases female fitness but there is no heritable variation for pathogen resistance.

4. While showy individuals may exhibit increased disease resistance, there should be cases where they do not (for the same reasons as in point 3, again in contrast to the good genes hypothesis, which posits a strong link). Few tests of actual pathogen resistance of showy males have been performed, even though this would seem to be a central prediction of the good genes theory.

5. If the main contribution to fitness is from reducing pathogen transmission and not from genetic factors (i.e., biological resistance is ineffective), then species with the highest parasite 
loads should have the brightest feathers (or other markings), as has been observed in jungle fowl (Hamilton and Zuk, 1982). We believe that the good genes theory should make the opposite prediction if good genes are linked to showy traits (i.e., it should predict that the showiest species should be the healthiest).

6. The theory assumes that showy traits should have originated as exaggerations of normal traits that are indicative of good health, but that the particular traits that occur may be arbitrary. This assumption would explain the huge diversity of secondary sex traits (tail feathers; wattles; crests) and behaviors (calls; dances; creation of bowers). These predictions seem to be borne out but require closer scrutiny.

7. The model predicts that both humble and showy types should be found to occur together if costs and benefits are in a proper balance. Such cases are known to exist and are not explained by good genes models.

Overall, this model makes a number of specific predictions. All of the predictions for which data exist seem to be supported, although data are sketchy at this time. Relatively simple experiments are possible to test whether female avoidance of sick individuals is enhanced by showy traits and whether showy males are actually more disease resistant.

We therefore conclude that the pathogen avoidance theory of sexual selection has a number of advantages. It can be directly related to risks of pathogen transmission in various populations and makes a specific prediction that species living in large groups should not exhibit showy traits because the traits do not reduce risk to the choosy female. The theory explains the origin both of the showy traits themselves as exaggerations of signs of good health, and the initial preference of females for showier males in terms of preexisting tendencies to avoid diseased individuals. The underlying model is operative when transmission risk is high, even in the absence of heritable variation for pathogen resistance traits, but will lead to selection for resistance traits if they are 
present. The model should be effective for both polygynous species and monogamous species. If the risk of disease is sufficiently high, the model may lead to showy traits in males and choosiness traits in females (although we have not shown this here). Finally, the model predicts that showy and humble types may coexist, a situation that has been observed in the field. Overall, the model resolves difficulties with good genes models and makes specific new predictions.

\section{ACKNOWLEDGMENTS}

This research is supported through contract W-31-109-ENG-38 with the U.S. Department of Energy, Office of Energy Research, Office of Health and Environmental Research, Program for Ecosystem Research. We thank V. Passekov for a helpful review of the manuscript. 


\section{REFERENCES}

Edwards, J. C. 1988. The effects of Trichinella spiralis infection on social interactions in mixed groups of infected and uninfected male mice. Animal Behavior 36:529-540.

Edwards, J. C. and C. J. Barnard. 1987. The effects of Trichinella infection on intersexual interactions between mice. Animal Behavior 35:533-540.

Hamilton, W. D., and M. Zuk. 1982. Heritable true fitness and bright birds: a role for parasites? Science 218:384-387.

Hart, B. L. 1987. Behavior of sick animals. Veterinary Clinics of North America: food Animal Practice 2:383-391.

Hart, B. L. 1990. Behavioral adaptation to pathogens and parasites: five strategies. Neuroscience and Biobehavioral Reviews 14:273-294.

Hart, B. L., and C. M. Haugen. 1971. Prevention of genital grooming in mating behavior of male rats (Rattus norvegicus). Animal Behavior 19:230-232.

Hart, B. L., E. Korinek, and P. Brennan. 1987. Postcopulatory genital grooming in male rats: prevention of sexually transmitted infections. Physiology and Behavior 41:321-325.

Hill, G. E. 1995. Ornamental traits as indicators of environmental health. BioScience 45:25-31.

Hofbauer, J., and K. Sigmund, K. 1988. The Theory of Evolution and Dynamical Systems. Cambridge University Press, Cambridge. 
Kennedy, C. E. J., . A. Endler, S. L. Poynton, and H. McMinn. 1987. Parasite load predicts mate choice in guppies. Behavioral Ecology and Sociobiology 21:291-295.

Kirkpatrick, M. 1986. Sexual selection and cycling parasites: a simulation study of Hamilton's hypothesis. Journal of theoretical Biology 119:263-271.

Kirkpatrick, M. 1989. Sexual selection: is bigger always better? Nature 337:116-117.

Loehle, C. 1995. Social barriers to pathogen transmission in wild animal populations. Ecology $76: 326-335$.

Maynard Smith, J. 1974. The theory of games and the evolution of animal conflicts. Journal of theoretical Biology 47:209-221.

Maynard Smith, J. 1982. Evolution and the Theory of Games. Cambridge University Press, Cambridge.

Mulvey, M., and J. M. Aho. 1993. Parasitism and mate competition: liver flukes in white-tailed deer. OIKOS 66:187-192

Pohley, H.-J., and B. Thomas. 1983. Nonlinear ESS-models and frequency dependent selection. Biosystems 16: 87-100.

Rätti, O., R. Dufva, and R. V. Alatalo. 1993. Blood parasites and male fitness in the pied flycatcher. Oecologia 96:410-414.

Read, A. F. 1988. Sexual selection and the role of parasites. Trends in Ecology and Evolution 3:97-102. 
Service, M. W. 1986. Blood-Sucking Insects: Vectors of Disease. Edward Arnold, London.

von Schantz, T., M. Grahn, and G. Göransson. 1994. Intersexual selection and reproductive success in the pheasant Phasianus colchicus. American Naturalist 144:510-527.

Witter, J. F. 1981. Brucellosis. pages 280-287, in Davis, J. W., L. H. Karstad and D. O. Trainer, D. O. (eds.). Infectious Diseases of Wild Animals. Iowa State University Press, Ames.

Yuill, T. M. 1981. Myxomatosis and fibromatosis. pages 154-177, in Davis, J. W., L. H. Karstad and D. O. Trainer (eds.). Infectious Diseases of Wild Animals. Iowa State University Press, Ames. 


\section{Figure Legend}

Figure 1. Time trajectories of the microevolution model (1-2.3) with parameters:

$$
G=1, H=2, S=0.15 \text {. }
$$




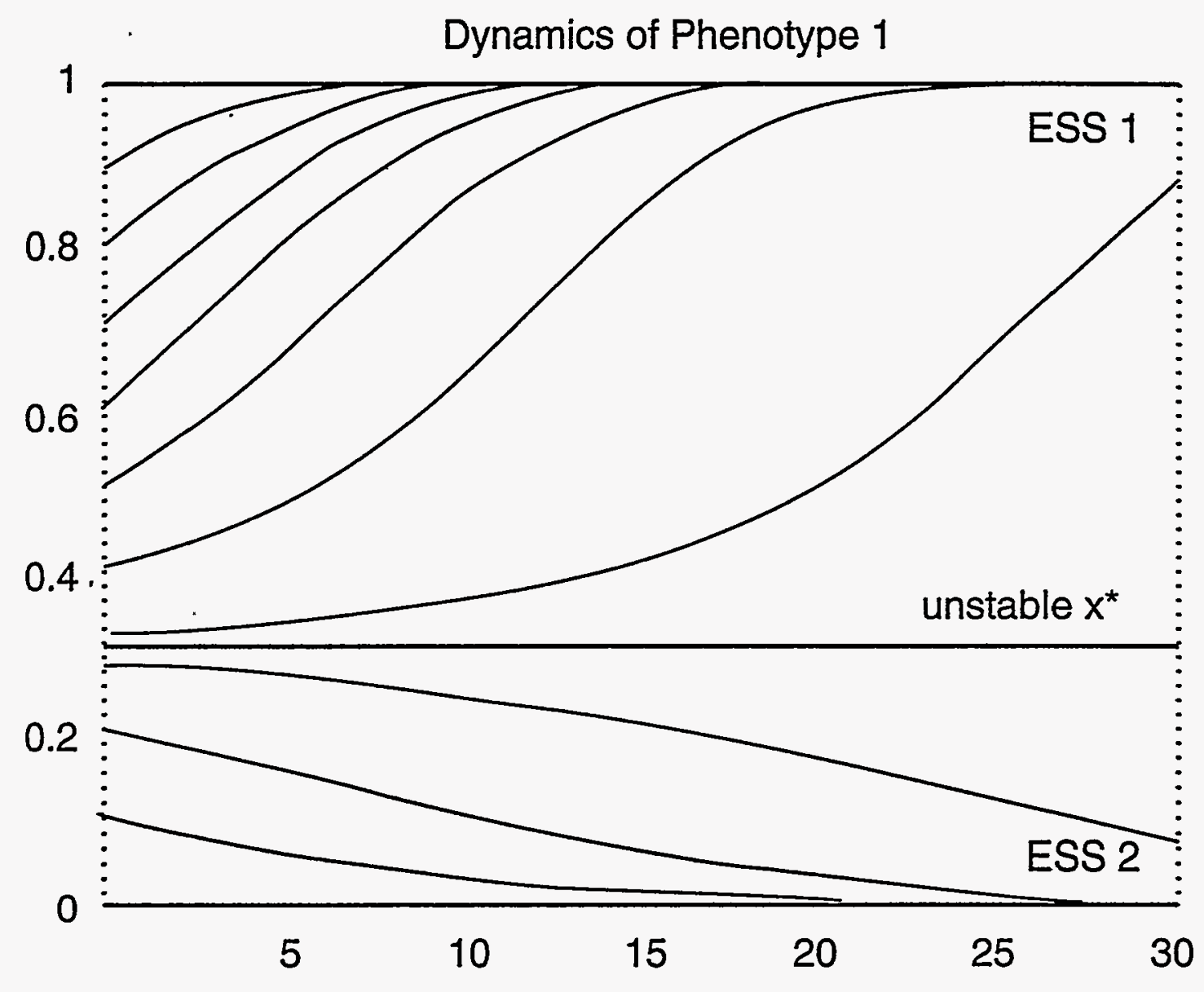


Table 1. Existence and stability of equilibria in equation (1.3)

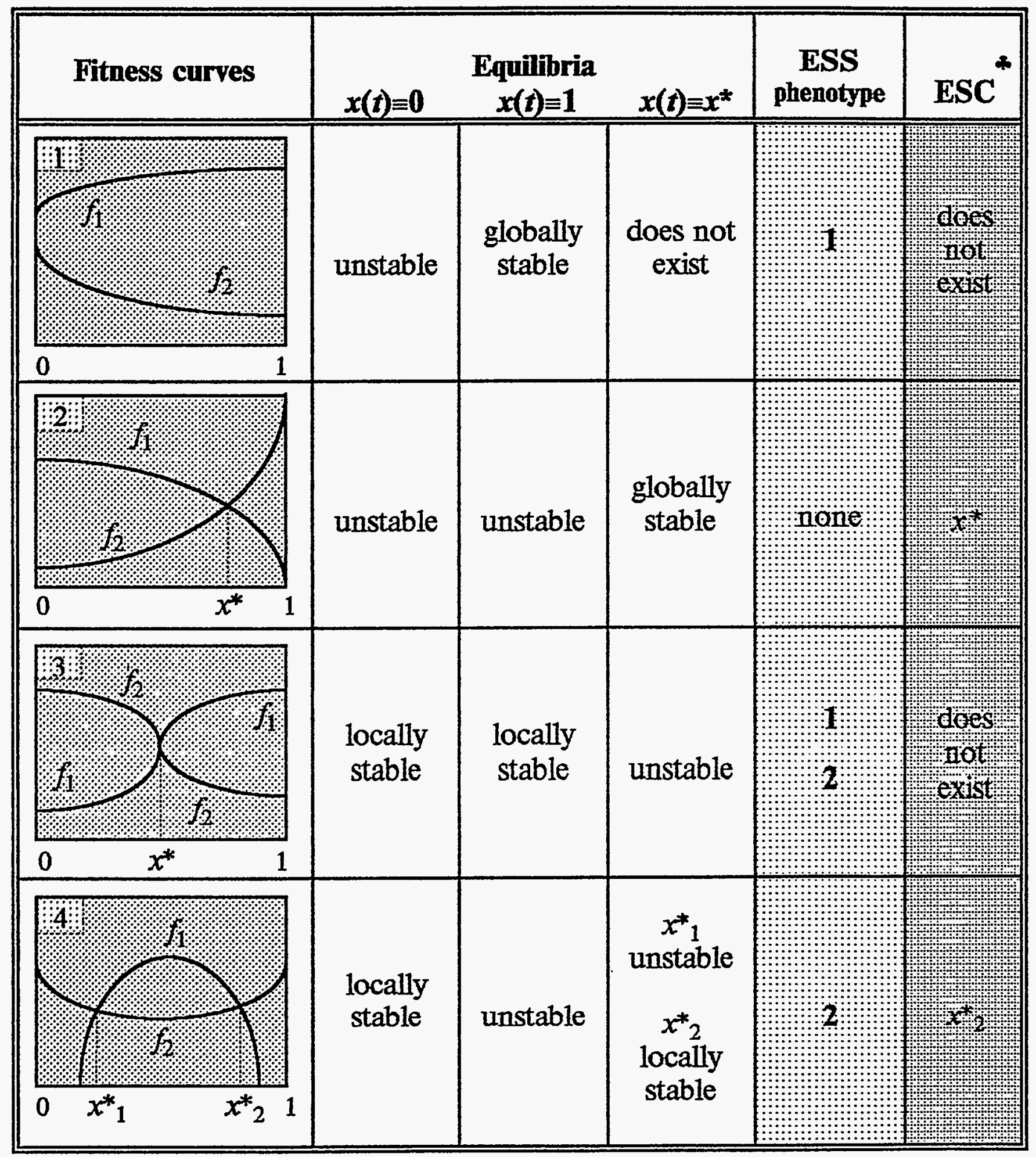

*) Homogeneous states are not regarded as "compositions". 
Table 2. Patterns of linear fitness functions, their ranges in the parameter space, and the outcome of microevolution

\begin{tabular}{|c|c|c|}
\hline Fitness lines & $\begin{array}{l}\text { Formal conditions } \\
\text { in terms of } f_{i}(0,1) \\
\text { and } G, H, S \\
\end{array}$ & $\begin{array}{l}\text { Parameter } \\
\text { in the 3-D }\end{array}$ \\
\hline 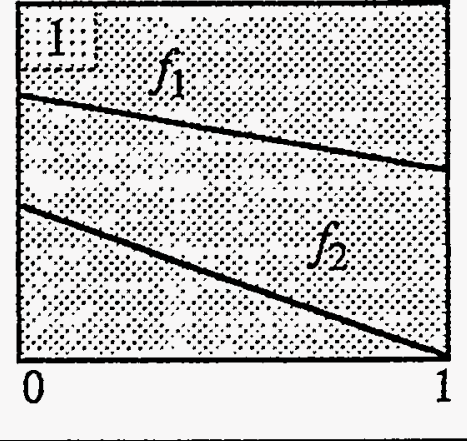 & $\begin{array}{c}f_{1}(0)>f_{2}(0), \\
f_{1}(1)>f_{2}(1) \\
S<\min \{G-H / 2, G / 2\}, \\
G>H / 2 . \\
\text { UnugueESS }\end{array}$ & $\begin{array}{l}\text { Below } \\
\text { the most } \\
\text { shaded } \\
\text { petals }\end{array}$ \\
\hline ?. & $\begin{array}{c}f_{1}(0)<f_{2}(0), \\
f_{1}(1)<f_{2}(1) \\
S>\max \{G-H / 2, G / 2\}, \\
G, H \geq 0 \\
\text { Unique ESS } \\
\text { Giamble? }\end{array}$ & $\begin{array}{l}\text { Above } \\
\text { the most } \\
\text { shaded } \\
\text { petals }\end{array}$ \\
\hline ?. & $\begin{array}{c}f_{1}(0)>f_{2}(0) \\
f_{1}(1)<f_{2}(1) \\
G / 2<S<G-H / 2, \\
G>H . \\
\text { Unique ESC: } \\
\text { Eshowy }\end{array}$ & $\begin{array}{l}\text { Between } \\
\text { the most } \\
\text { shaded } \\
\text { petals }\end{array}$ \\
\hline ? & 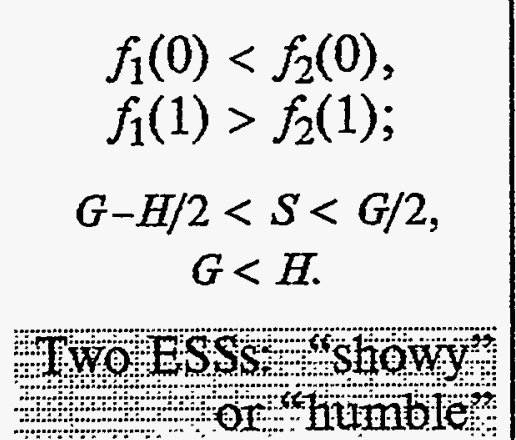 & $\begin{array}{l}\text { Between } \\
\text { the most } \\
\text { shaded } \\
\text { petals }\end{array}$ \\
\hline
\end{tabular}


Table 3. Outcomes of microevolution in the populations where females can discriminate between sick and healthy males and where they cannot.

\begin{tabular}{|c|c|c|c|c|c|c|}
\hline \multirow{3}{*}{$\begin{array}{c}\text { Can } \\
\text { or } \\
\text { cannot } \\
\text { disri- } \\
\text { minate }\end{array}$} & \multicolumn{6}{|c|}{ Formal parameters } \\
\hline & \multirow[b]{2}{*}{$G$} & \multirow[b]{2}{*}{$H$} & \multicolumn{3}{|c|}{$S$} & \multirow[b]{2}{*}{$\begin{array}{l}\text { Case 1': } \\
\text { Plumbie? } \\
\text { tuss }\end{array}$} \\
\hline & & & Case 1: & 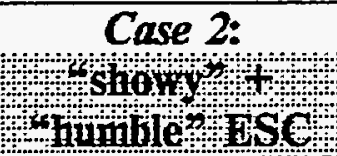 & 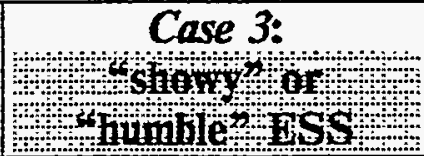 & \\
\hline $\begin{array}{c}\text { non- } \\
\text { discrim. }\end{array}$ & $H_{0}$ & $H_{0}$ & $S_{\mathrm{nd}}<0.5 H_{0}$ & \multicolumn{2}{|c|}{$\begin{array}{l}\text { Impossible as separate cases, } \\
\text { but degenerate at } S_{\text {nd }}=0.5 H_{0} \\
\text { to the "no difference" case }\end{array}$} & $S_{\mathrm{nd}}>0.5 H_{0}$ \\
\hline $\begin{array}{c}\text { non- } \\
\text { discrim. }\end{array}$ & $\begin{array}{c}0.8 \times \\
H_{0}\end{array}$ & $H_{0}$ & $S_{\mathrm{nd}} \leq 0.3 H_{0}$ & $\begin{array}{c}\text { Impossible } \\
(\text { since } G<H)\end{array}$ & $0.3 H_{0}<S_{\mathrm{nd}}<0.4 H_{0}$ & $S_{\mathrm{nd}} \geq 0.4 H_{0}$ \\
\hline discrim. & $2 H_{0}$ & $H_{0}$ & $S_{\mathrm{d}} \leq H_{0}$ & $H_{0}<S_{\mathrm{d}}<1.5 H_{0}$ & $\begin{array}{c}\text { Impossible } \\
(\text { since } G>H)\end{array}$ & $S_{\mathrm{d}} \geq 1.5 H_{0}$ \\
\hline discrim. & $4 H_{0}$ & $H_{0}$ & $S_{\mathrm{d}} \leq 2 H_{0}$ & $2 H_{0}<S_{\mathrm{d}}<2.5 H_{0}$ & $\begin{array}{c}\text { Impossible } \\
(\text { since } G>H)\end{array}$ & $S_{\mathrm{d}} \geq 2.5 H_{0}$ \\
\hline
\end{tabular}

